\title{
Publisher's Note: Unbinding slave spins in the Anderson impurity model
}

[Phys. Rev. B 96, 201106(R) (2017)]

Daniele Guerci and Michele Fabrizio

(Received 17 November 2017; published 27 November 2017)

DOI: 10.1103/PhysRevB.96.199905

This paper was published online on 13 November 2017 with typographical errors in creation-operator-annihilation-operator products throughout the Rapid Communication. These terms have been corrected as of 15 November 2017. These terms are correct in the printed version of the journal. 